\title{
Methodology for Innovation-Based Control of Business Changes Taking into Consideration the Communication Aspects
}

\author{
Khuta Gumba ${ }^{l}$,Svetlana Uvarova ${ }^{2}$, Svetlana Belyaeva ${ }^{2}$ and Svetlana Revunova ${ }^{1, *}$ \\ ${ }^{1}$ Moscow State University of Civil Engineering, Yaroslavskoe shosse, 26, Moscow, 129337, Russia \\ ${ }^{2}$ Voronezh State Technical University, 14, Moskovsky prospect, Voronezh, 394026, Russia
}

\begin{abstract}
At the current stage of the economy development the efficient control of the enterprise activity based on the anticipatory adaptation to continuous changes of the environment in tune with the trend of the internal medium dynamic self-organization is the most important prerequisite of the success, so the issues of scientifically substantiated control of changes become especially urgent. The authors have substantiated the essence and comprehensive nature of the process of business changes, and its predominantly innovative character has been determined. The algorithm of controlling business changes at the enterprise based on graph theory methodology has been suggested based on identification of the communication basis of business processes. Using the algorithm enables making scientifically substantiated decisions facilitating achievement of the enterprise functioning goals at some period of time. Based on the algorithm, the authors have developed methodology for optimization of innovation-based organizational enterprise management structure implemented in business practice of RZhDstroi JSC Holding. The results of the suggested methodological tooling validation have shown the appropriateness of the tools in the enterprise business activity.
\end{abstract}

\section{Introduction}

The current research works in the field of efficient organization of economic activity associate organizational dynamics with processes of economic changes the common basis for which is innovations. The process of continuous anticipatory adaptation to the external spontaneous changes makes it necessary to generate some new methods of solving economic, organizational and structural and institutional problems of the enterprise development. Therefore, a task of scientific substantiation of essence and process of business changes and development of methodological tools and practical recommendations for enhancing efficiency of innovation-related organizational changes having innovative genesis becomes appropriate.

*Corresponding author: svr40889@mail.ru 
However, the existing theoretical and methodological resources for managing organizational changes are insufficient for implementation of tuned management in respect of provision of consistent development of both individual enterprises and the whole country economy.

The communicative nature of organizational processes is reflected in specific features of managerial innovations, which should be taken into consideration during study of the organizational changes phenomena. The necessity of the development of objective methodological tools for assessing the efficiency of organizational innovations at enterprises makes the article topical in respect of business practice.

\section{Methods}

In terms of the procedural approach to management, the change process element is a significant attribute of the concept of organizational innovation. Based in retrospective analysis $[1,6,10$, etc.], the following key elements were identified during determination of the concept "change":

1) the state opposite to stability;

2) movement and interaction of system elements;

3) processes of various nature based on the attributes of reversibility, focus and initialisability;

4) permanence of the change process;

5) change of the qualitative state which can be expressed quantitatively.

It should be noted that the change of the system as a whole occurs when a system element changes if there is some relationship forming the system structure, that is the organized state or order.

Only if this principle is fulfilled, the system can become an organizational system which needs the organization process, that is management for appearance of the property of organization [2]. Thus, the organizational changes shall mean changes of business subsystem of the enterprise (based on subjective attribute), the process of transforming of the structural elements order, their substantial and personal relations and relationships concerning management of the enterprise system elements.

Functioning of organizational systems is impossible without presence and development of economic mechanisms, which forms the principle of complexity of changes establishing the necessity of continuous invariance of forms and methods of economic incentive during transformation of organizational relationships [3, 4].

In addition, any change of economic methods of management aids in creation and change of institutional structures $[5,6]$.

Thus, the principle of complexity of changes postulates the necessity of joint changes economic, organizational and institutional changes in the aggregate, tuned with other change types for achievement of the set goals. The aggregate of all types of changes forms the life cycle of business changes (BC) (Fig. 1). 


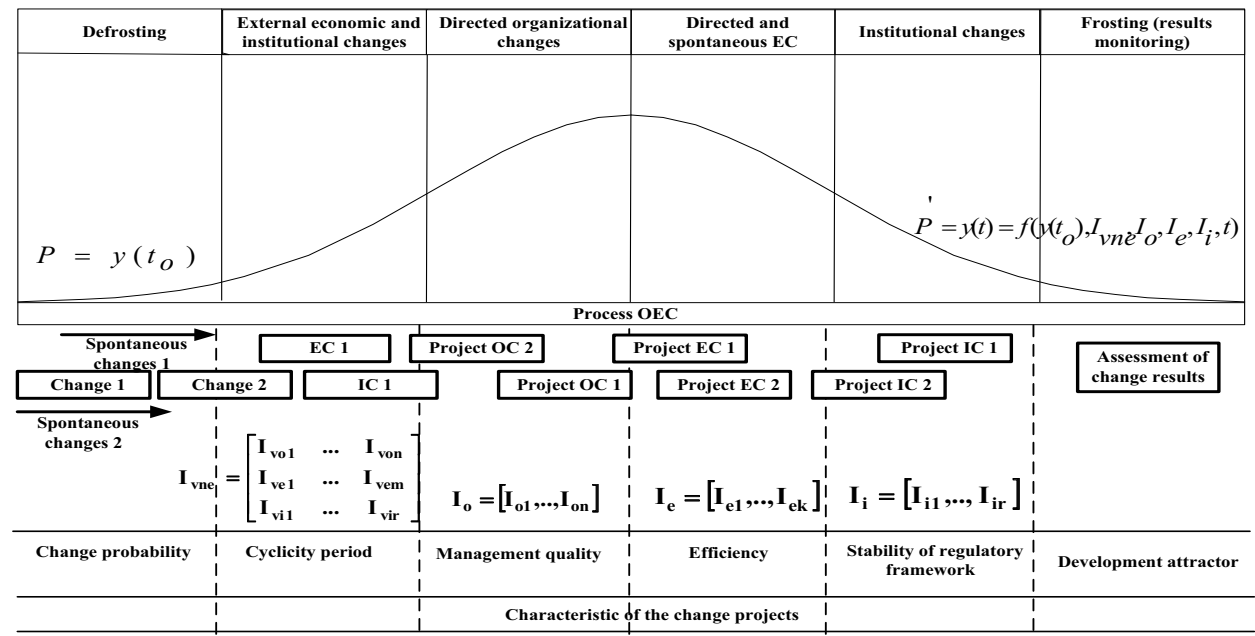

Fig. 1. Model of BC life cycle

where $\mathbf{I}_{o}-$ is the vector of organisational changes (OC), $\mathbf{I}_{e}-$ is the vector of economic changes (EC), $\mathbf{I}_{\mathbf{i}}-$ is the vector of institutional changes (IC), $\mathbf{I}_{\mathrm{vne}}-$ is the vector of external (spontaneous) changes, $\mathbf{t}-$ is time, $\mathrm{y}$ is the enterprise state change process, $\mathrm{P}$ is the initial state of the enterprise management system state, $\mathrm{P}^{\prime}$ - changed state of the enterprise management system

Taking into consideration methodological principles and approaches, and the results of semantic analysis of the concept "changes", based on correlation of business system elements allowing influencing the organization process (i.e. managing), the business changes shall be treated as an alternative to stability process of continuous movement and interaction of organizational and economic links between the system elements resulting in the measured change of qualitative state of both business subsystem and the whole system, and characterised by properties of reversibility, focus and initialisability.

As shown by scientific research works and practical developments, the business changes are innovation-related, since engineering and process innovations are the driver of the external and internal environment [7,9]. At the same time, innovations are a mandatory element of objective tree of any modern enterprise. They allow enhancing the product quality and creating stable competitive advantages. The semantic analysis enables identifying the replacement of old with new items, that is the change as a significant attribute $[8,9]$. This conclusion substantiates the possibility and expediency of applying the methodology in planning and implementation of business changes to innovation development and implementation process. Following the results of semantic analysis of innovation concept and the business change life cycle which we determined, it is innovations that initiate and catalyse the business changes, simultaneously being changes in essence and being one of the predominant objectives of the changes (Fig. 2).

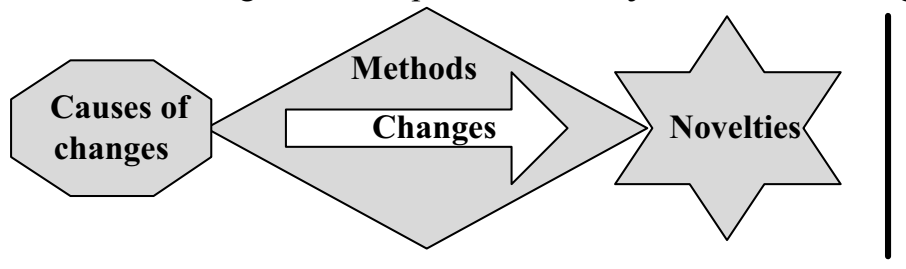

organizational marketing technological

Fig. 2. "Changes-innovations" contour 
However, it shall be noted that not any novelty facilitates the change of the state of the whole organizational system or its element [10]. Moreover, the organizational changes not always result in novelties, since not any organizational change has significant attributes of an innovation. [11]. Although, taking into account that organizational innovations can be borrowed, almost any organizational change for the particular enterprise will be innovative. In this case, taking into consideration the system character of the enterprise, the links between the system elements are presented as changes characterised by possibility to form a novelty, that is innovation.

On the other hand, the enterprise is a system the subsystem activity integration of which is carried out using communications objectively typical for purposeful activity implemented by integration of personnel and resources. Communications are a process of interaction between the system elements, system and the external medium based on the interests, incentives and values [12]. Therefore, it is expedient to consider the organizational changes, including innovative ones, in terms of communication theory.

The communication field of the enterprise is formally an oriented graph, the communication process participants are assumed to be the events of it, and transaction costs of data transmission are the characteristics. The objective of the organization functioning determines the objective function of the graph formalised using the method of distances.

The general algorithm of managing organizational changes at the enterprise taking into consideration communication aspects is shown as a flow chart (Fig. 3).

To identify and assess any communication issues, we deem it necessary to use the calculation and expert methodology. Its first stage is the use of expert evaluation method to identify and evaluate the importance of communication issues, and the second stage is carrying out of the design validation using the transaction cost estimate methodology. If there are apparent communication field issues accompanied by high transaction costs, it becomes necessary to change the communication field in accordance with the organizational solution objectives.

As applied to the organizational innovation process, it can be concluded that the innovations can be implemented both in determination of the communication participants and during data transmission and exchange. In this case it is necessary to identify novelties in external and internal communications; in organization of communication space (availability and quantity of vertical and horizontal communications and their assemblies); in formal communications (including those aimed at optimisation of non-formal communications, for example, overcoming resistance to changes); as a data transmission process (decrease of the corrupted data amount and increase of data transmission rate).

To develop the efficient organizational novelties, we suggested modifying the activity progress scheduling methodology during presentation of communication field of the enterprise taking into consideration elements of models of teams and groups, models of organisational structures.

The graph can also be optimised in terms of data transmission time (to decrease the critical path duration) or path length (to decrease the quantity of those engaged in communications by changing the structural communication module composition). The communication intensity, that is the amount of the information being transmitted and the quantity of functions being carried out also influence the information reliability. 


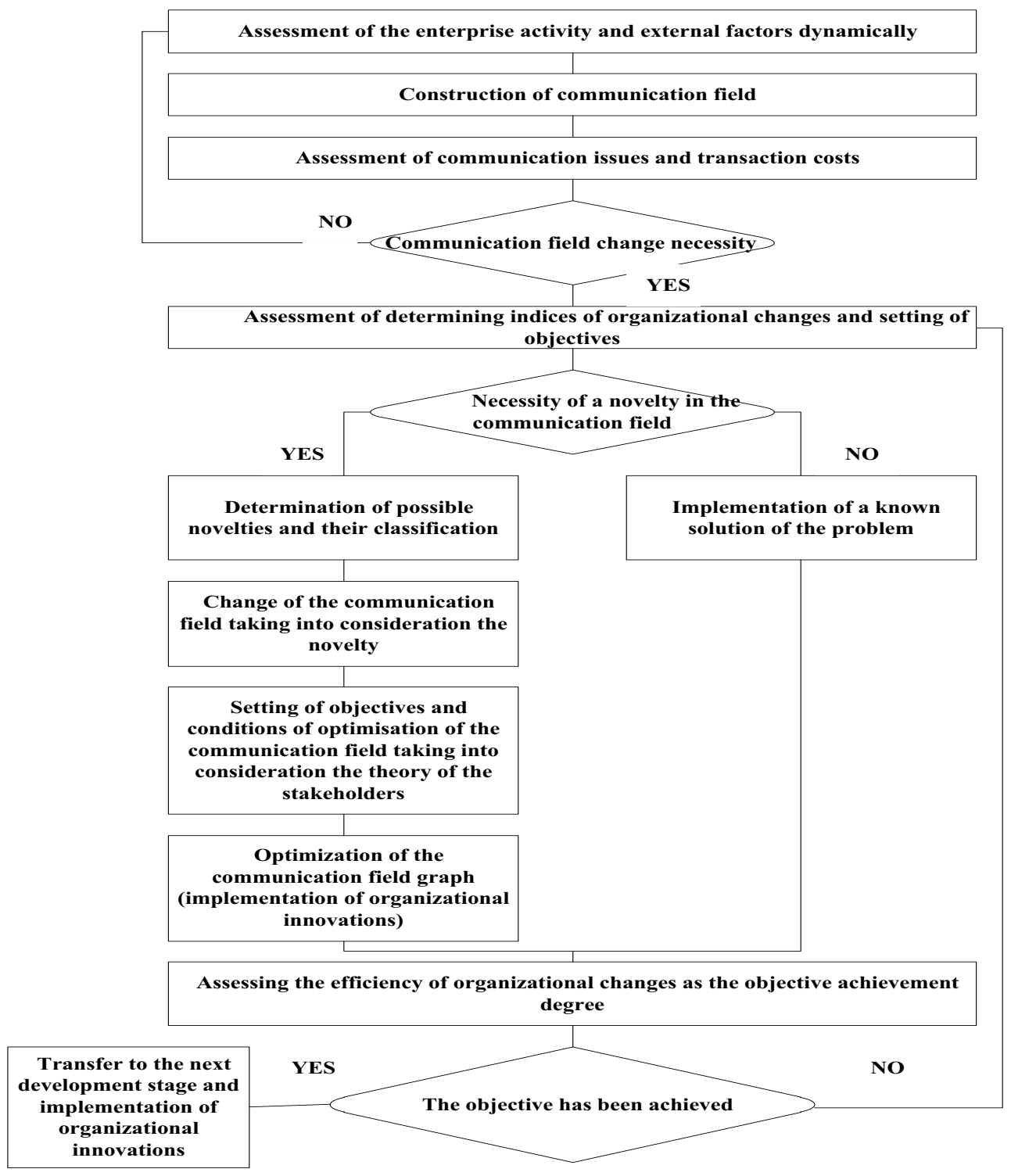

Fig. 3. The flow chart of the algorithm for managing organizational changes at the enterprise

We deem it possible and expedient to optimise the communication graph on transaction costs so that to minimise them and find the critical path on the graph (similarly to time optimization in project management system [12]) as the basis of improving innovations:

$$
\begin{gathered}
I=\sum_{i \in R_{i}} \mathrm{TИ}_{i j} \rightarrow \min \\
\boldsymbol{t}_{\text {кр }} \leq \mathrm{T} \\
\sum_{\boldsymbol{i} \in \boldsymbol{R}_{\boldsymbol{i}}} Д_{i j} \geq \text { Д }_{\text {min }}
\end{gathered}
$$


where $I$ is the total transaction costs; Tuij is transaction costs of transmission from vertex $i$ to vertex $j$; Ri is the quantity of intermediate links (for aggregated graph); Дij is the information reliability degree, $Д_{\min }$ is the minimum admissible information reliability degree, tкp is the critical path of the graph (in terms of duration).

Also, taking into consideration the nature of communications, we deem it possible to optimise the oriented graph in terms of the efficiency criterion [2,10] (based on profit dynamics, profitability of the activity or production):

$$
\begin{aligned}
\boldsymbol{K}(\boldsymbol{T})= & \frac{\sum_{\boldsymbol{T}} \ni_{\boldsymbol{i j}}}{\sum_{\boldsymbol{T}} \mathrm{TИ}_{i j}} \rightarrow \boldsymbol{m a x} \\
& \boldsymbol{t}_{\text {кр }} \leq \mathrm{T} \\
& \sum_{\boldsymbol{i} \in \boldsymbol{R}_{\boldsymbol{i}}} Д_{\boldsymbol{i}} \geq Д_{\min }
\end{aligned}
$$

where $Э i j$ is the communication outcome $i-j$ characterised by the profit margin or break-even level.

Type of optimisation, objective function and limitations will be determined by specificity of the identified issue and its solution aims. The optimised communication graph shall ensure the maximum achievability of the set objective of organizational changes on the innovative basis.

\section{Results}

The organization structure of the enterprise influences the information quality, and information corruption during transmission and rate of transmission via data channels. The more efficient is the communication process at the enterprise, the prompter it reacts to the market changes associated with improvement of the production processes and, consequently, the growth of innovation activity. The significance of the enterprise management organisation structure forms the necessity of the structure optimisation implemented on the innovative basis (Fig. 4).

The algorithm validation was carried out during solution of the task of changing the organisation structure of construction and installation business unit No. 7 of RZhDstroi JSC. Low performance of the construction holding division caused by unnecessary existence of some structural units and high administrative expenses makes it necessary to implement innovation-based organizational changes.

Using the transaction costs estimation methodology we offered [12], loaded communication graph was plotted (Fig. 5). It shall be noted that due to impossibility to carry out process accounting at the enterprise, it is only possible to calculate the parameters of the aggregated communication graph of the enterprise organisation structure for aggregated communication process "execution of construction and installation works".

The results of analysis of the aggregated communication graph for the main function of the enterprise allow concluding that the organisation structure centralisation degree is rather low, at the same time, the management density level is high, and the coefficient of adequacy for the job is high. According to the calculations performed, the maximum transaction costs of the communication process at the set probability level of $95 \%$ amount to $23,428.37$ thous. roubles. All communications are redundant, which precludes overloading of structural units of the communications.

To assess the efficiency of the organizational innovation (which is transfer of the most part of the performed works to outsourcing with respective reduction of loss-making structural units and grouping of divisions in accordance with the updated functions) implemented on the management's initiative, a similar aggregated communication graph 
was plotted for the new organisation structure of construction and installation business unit No. 7 (Fig. 6).

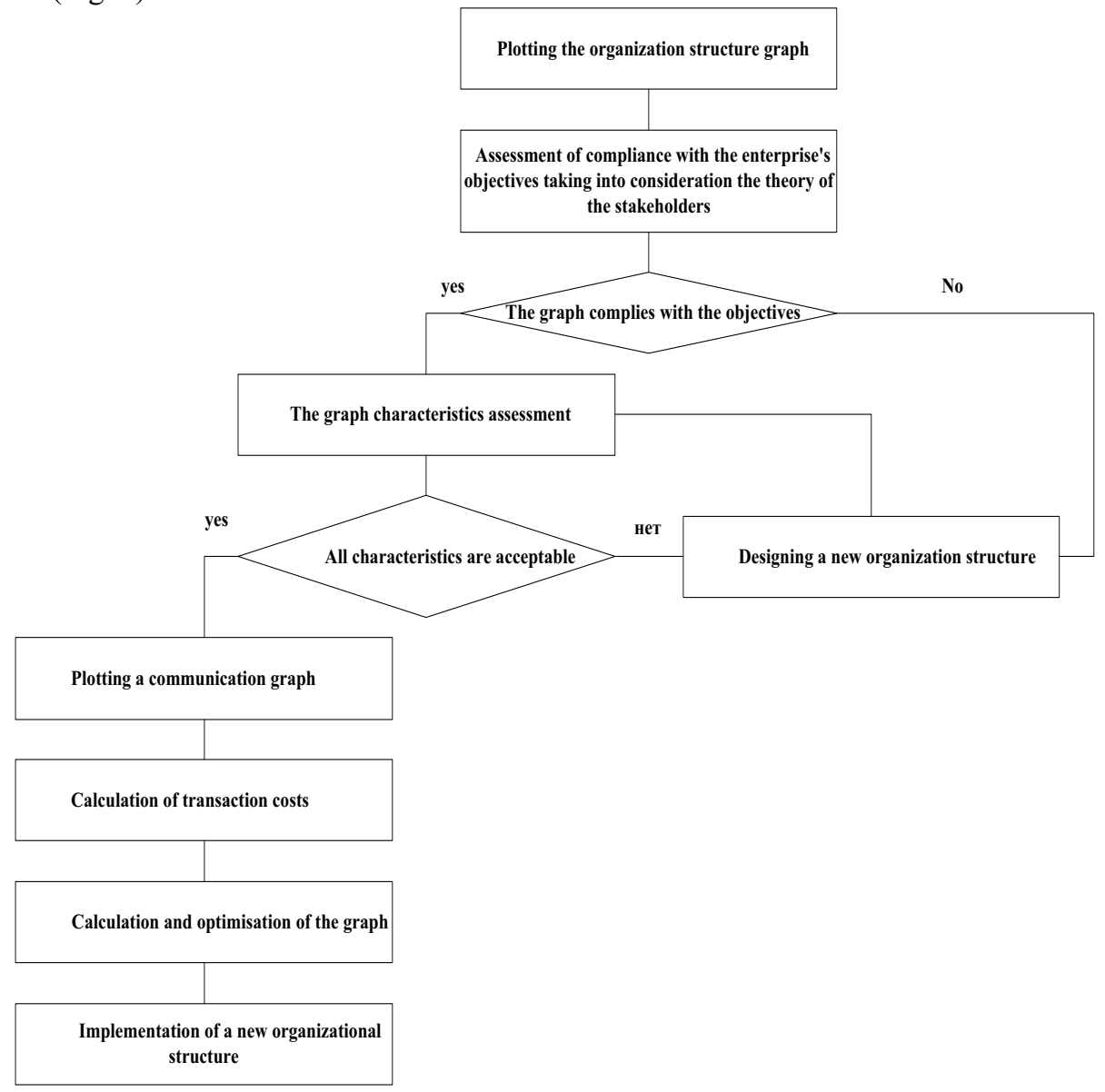

Fig. 4. The flow chart of the innovation-based organisation structure optimisation algorithm

The results of analysis of the aggregated communication graph for the main function of the enterprise allow concluding about increase of the organization structure centralisation degree, simultaneous decrease of the management density (due to reduction of structural units), decrease of the coefficient of adequacy for the job. The organizational innovation suggested by the management of Russian Railways JSC, despite all its advantages, confines the functionality of construction and installation business unit No. 7 to execution of the role of the general contractor exclusively, the aggregated communication graph of the main communication process having the only path (being the critical path), which does not match the criteria of non-manipulated mechanisms and results in impossibility to optimise the communications. 


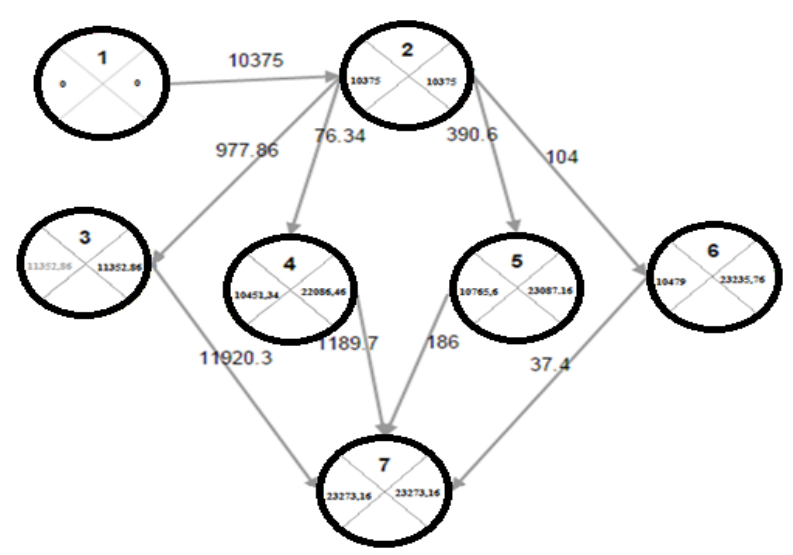

Fig. 5. Oriented graph of the organization structure of SMT-7 of RZhDstroi JSC for aggregated communication process "execution of construction and installation works"

Where 1 is the customer (Russian Railways); 2 is the management apparatus of construction and installation business unit No. 7; 3 is the bridge construction train; 4 is the specialised construction and installation train; 5 is the reinforced concrete production plant; 6 is subcontractors; 7 is RZhDstroi JSC Holding

Such organizational change, provided the administrative costs are reduced, has some negative consequences: decrease of the interest of the enterprise's labour collective and management in the activity results (respectively, the growth of transaction costs for monitoring of execution of duties by employees); emergency of possibilities to manipulate both on the part of the business unit and on the part of the parent company (which results in the growth of costs on elimination of behavioural uncertainty associated with monitoring of the subsidiaries' activity and overcoming of the information asymmetry); possibility of manipulation by outsourcers (causing growth of transaction costs for monitoring and quality, as well as other contracting costs, necessity of growing costs for creation of legal framework); emergency of resistance to any changes by the personnel due to job cuts (and the respective growth of transaction costs for overcoming of opportunist behaviour); excessive management apparatus (transaction costs for functioning of the management apparatus can be reduced as a whole, but simultaneously with such costs efficiency decrease).

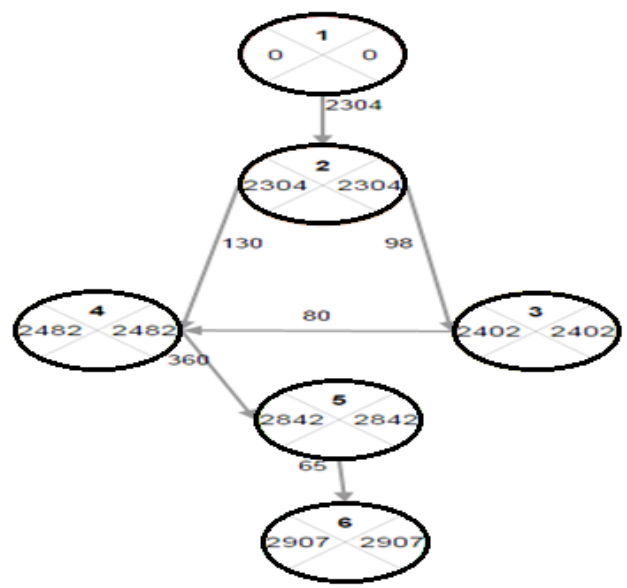

Fig. 6. Communication field (aggregated loaded oriented graph) of the innovative organization structure of construction and installation business unit No. 7 for the aggregated communication process "execution of construction and installation works" 
where 1 is the customer (Russian Railways); 2 is the management apparatus of construction and installation business unit No. 7; 3 is economic service of construction and installation business unit No. 7; 4 is engineering service of construction and installation business unit No. 7; 5 is subcontractor organisations; 6 is RZhDstroi JSC Holding.

Due to the change of the external medium expressed in increase of load on the business unit caused by construction of "Zhuravka - Millerovo" railway (a route to bypass the Ukraine), it is necessary to revise the solution on organizational changes of the structure of construction and installation business unit No. 7 based on optimisation of the initial structure of management. The cost of the new project is 56.6 billion roubles, including 7 billion roubles to be spent in 2015 . The length of the railway is $136.9 \mathrm{~km}$. The construction works on this railway are scheduled to be completed in 2017. Despite increase of the scope of works in prospect, we believe that at low performance of the business unit the strategic goal shall be substantiated not by the growth of the scope of works, but by the growth of the works implementation profit. In this case it is necessary to meet the condition of minimising the transaction costs and maximising the provision of the enterprise with agreements and capacity utilisation (according to conditions of communications efficiency). Taking into consideration that construction and installation business unit No. 7 is a subsidiary of RZhDstroi JSC and the load is determined by the central office of the holding, the parameters of provision with agreements and capacity utilisation can be neglected.

To determine a more efficient organizational innovation, we have constructed, analysed and optimised the communication graph of the organisation structure on the efficiency criterion. For the construction and installation business unit No. 7 the efficiency was determined as a ratio of the profit from execution of construction and installation operations to transaction costs of the respective communication process. As a result, the optimum organization structure of construction and installation business unit No. 7 management was obtained by liquidation of one structural subdivision - reinforced concrete production plant (Fig. 7).

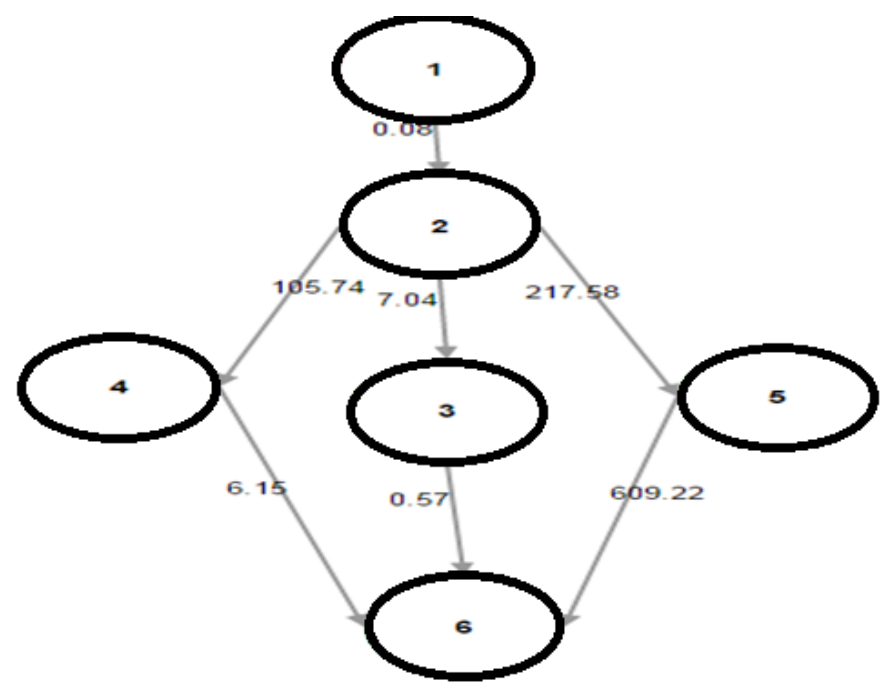

Fig. 7.The optimised communication graph of liquidation of construction and installation business unit No. 7 organisation structure for the aggregated communication process "execution of construction and installation works" on the efficiency criterion.

where 1 is the customer (Russian Railways); 2 is the management apparatus of construction and installation business unit No. 7; 3 is the bridge construction train; 4 is the specialised construction and installation train; 5 is subcontractors; 6 is RZhDstroi JSC Holding 


\section{Discussions}

Analysing the calculation results, it can be concluded that the transaction costs in the optimised graph are reduced as compared with the initial graph (by means of liquidation of one structural element. However, as compared with the innovative structure graph, all costs we identified, associated with restructuring are decreased) which influenced the communication efficiency growth. As a result of the communication graph optimisation, it was found out that implementation of optimised structure of construction and installation business unit No. 7 is the most efficient solution. In this case the organization's objectives will be achieved to the maximum extent: the total profit will amount to $365,304.83$ thous. roubles, which will result in optimisation of the enterprise objective (based on the target profit margin of 370,000 thous. roubles).

\section{Conclusion}

The tendency of increasingly high role of managerial innovations in the organization development has been topical in the recent years; in this case the information managerial innovations shall be separated specially - information systems and technologies which include managerial decision-making support software, application packages for automation of economic activity, etc.

The process of diffusion of managerial innovations in information sphere is highly dynamic, as the software is fully updated in rather short time, which allows promptly responding to change of managerial mechanisms aimed both at poorly-automated enterprises and corporations with high degree of business process automation and informatization.

Provided an automated process control and budgeting system is implemented in construction and installation business unit No. 7, just like in any other enterprise, it becomes possible to plot detailed communication graphs for each process, calculate transaction costs and carry out optimisation in accordance with the suggested methodological tools which, as proved by validation results, aids in increasing the enterprise performance efficiency.

It shall be noted that the results of validation of the suggested methodological tools have proved their topicality in the current business practice of the enterprises in terms of enhancement of both innovative and production and administrative performance.

\section{References}

1. M. A. Ageeva. Bulletin of the University (State University of Management). 8, (119118). 2011.

2. Kh. M. Gumba.Theoretical basis of innovative development of the construction industry, MGSU, Moscow, 2012.

3. V. S. Kankhva. Bulletin MGSU. 6, (161-166). 2011.

4. V. S. Kankhva. An international conference dedicated to the 100th anniversary of J. B. Monfreda, 2013

5. I. G. Meshcheryakov. Modern aspects of the economy. 10 (194), (41-47), 2013.

6. S. V. Rodionova,V. A. Vlasenko. Bulletin MGSU.9, (158-167). 2011.

7. S. V. Rodionova. Bulletin MGSU. 6, (131-139). 2015.

8. S. I. Sergeeva. Bulletin of Voronezh State Technical University, 5, (15-17), 2012. 
9. D. N. Silka,K. V. Urazova. Bulletin MGSU. 8. (171-185). 2015.

10. S. Y. Tyukova. Herald ENGECON. Series: Economy. T. 28. № 1. (96-100), 2009.

11. M. T. Hannan.Change Management: a reader.Publishing House of the Executive. wk. Management, St. Petersburg, 2010.

12. A.D. Ishkov, M.Yu. Mishlanova,K.P. Grabovyi. International Journal of Applied Engineering Research. T. 11. № 3. (1676-1679), 2016. 